\title{
Robust Nonlinear Adaptive Controller Design for Horizontal Position Control of a Rotary Wing Autonomous Vehicle Using Backstepping Method
}

\author{
Tushar Kanti Roy \\ Department of Electronics \& Telecommunication Engineering, Rajshahi University of Engineering \& Technology, Rajshahi, Bangladesh
}

\section{Email address:}

roy.kanti03@gmail.com

\section{To cite this article:}

Tushar Kanti Roy. Robust Nonlinear Adaptive Controller Design for Horizontal Position Control of a Rotary Wing Autonomous Vehicle Using Backstepping Method. Automation, Control and Intelligent Systems. Vol. 3, No. 6, 2015, pp. 104-111. doi: 10.11648/j.acis.20150306.12

\begin{abstract}
In this paper, a new approach of designing robust adaptive backstepping controller for horizontal position control of a rotary wing autonomous unmanned vehicle (RAUV) with consideration of parametric uncertainties and external disturbances is proposed. Based on this new approach, the proposed RAUV controller is adaptive to the parametric uncertainties and robust to the external disturbances. To prove the convergence of different tracking error to zero, a control Lyapunov function (CLF) is formulated in every step of the design process of controller and which is guaranted through the negative definiteness of the derivative of CLF. At last, a numerical evaluation is performed on a highly fedility nonlinear simulation model to justify the usefulness of the proposed controller. The performance of the designed controller is also compared with a classical PID controller. Simulation results demonstrate that the proposed controller provides an improved performance for the closed-loop system in the presence of parametric and external uncertainties within the UAH model over the existing controller.
\end{abstract}

Keywords: Adaptive Robust Backstepping Controller, Control Lyapunov Function, External Disturbance, Rotary Wing Autonomous Vehicle, Parametric Uncertainty

\section{Introduction}

There are variety of rotary wing unmanned autonomous vehicles (RUAV), but among these the unmanned autonomous helicopters (UAHs) constitute one of the most versatile and agile platforms for the development of autonomous flight systems. A small-scale unmanned helicopter can operate in different flight modes, such as vertically take-off/landing, hovering, longitudinal or lateral flight, and bank to turn which gives them the advantage of effective observation from various positions. Hovering and vertically take-off are necessarily needed. Nowadays, there are new trends of UAH controller design due to their high level of agility, maneuverability and capability of operating in adverse weather conditions. To achieve these performances of an $\mathrm{UAH}$, the trajectory tracking and disturbance rejection capability need to be significantly improved. But the problem is that it is a naturally unstable system with nonlinear dynamics. The main difficulties of an
UAH are higher nonlinearities which arise from the crosscouplings due to the tail rotor, main rotor, engine and dynamic uncertainties [1]. Besides, it is an underactuated mechanical system with six degrees of freedom (6-DOF) because it has only four control inputs. To cope such problems as mentioned above, different approaches have been proposed by the researchers in literature [2]-[4]. Thus, in order to improve the tracking performance of an UAH in the presence of parametric and external uncertainties within the system a robust adaptive backstepping controller is proposed in this paper.

Different conventional controllers are available to stabilize to flight of an UAH which are designed on the linear approximation around an operating point [5], [6]. But these controller are not suitable when operating point is changed due to any external or inter uncertainties. Thus, recently, various advance nonlinear control techniques have been applied to the control the UAH for different operating points under large disturbance [7]-[11]. A robust $\mathrm{H}_{\infty}$ control method of the longitudinal and lateral dynamics of the BELL 205 
helicopters in the presence of model uncertainties is presented in [12]. A robust feedback method is propoed in [13] to reject the external wind gust, where the external wind gust is assumed to be the sum of a fixed number of sinusoids with unknown amplitudes, frequencies and phases. To control of an autonomous scale helicopter under the consideration of parameter uncertainties and uniform time varying three-dimensional wind gusts a backstepping method is proposed in [14]. A nonlinear $\mathrm{H}_{\infty}$ horizontal position controller for hovering and landing flight of a RUAV in the presence of horizontal wind gusts is proposed in [15]. Similar control approach is used in [16] to control the altitude and attitude for a hovering flight of an UAH in the presence of vertical wind gusts. A nonlinear robust small-gain control method is proposed in [17] to control the vertical motion of an autopilot helicopter during landing flight condition in the presence of parametric uncertainties on the plant and actuator model. The hover flight control of a helicopter by considering the 1-DOF system using the neural adaptive technique was proposed in [18]. Though, these controller are designed by considering the uncertainties with the system of an UAH but how to control the longitudinal and lateral dynamics of an UAH are not clear in this papers. Moreover, the longitudinal and lateral dynamics control to improve the trajectory tracking performance of an UAH under consideration of parametric uncertainties along with external disturbances are still uncovered.

The main aim of this paper is to design a robust adaptive bacstepping controller to control the horizontal position of an UAH under the consideration of parametric uncertainties and external disturbances. Note that backstepping is a recursive nonlinear control design method, which provides an alternative to the feedback linearization. The advantage of this technique over feedback linearization technique is that it can gain from the stabilizing nonlinear terms rather than eliminating them. For the controlling purposes, the forward and sideward motions of an $\mathrm{UAH}$, together with roll and pitch motions are controlled by lateral and longitudinal cyclic stick inputs via the flapping motion of the main rotor. Finally, a nonlinear simulation model is used to evaluate the effectiveness of the designed controller for hovering flight control and compared to that of an existing PID controller.

The rest part of this paper is organized as follows. Section 2 briefly introduces the mathematical model of UAH to design the controller. The control problem is formulated in Section 3. Section 4 presents the design procedure of the proposed controller. Section 5 discusses the simulation results. Finally, the conclusion of the work is presented in Section 6.

\section{Dynamical Model of UAH}

The UAH has the specific characteristic as compared to fixed wing aircraft such as, it can move vertically, float in the air, turn in place, move forward and laterally and can perform these movements in combinations. Due to these characteristics, the dynamic modeling of an UAH is a very complex problem. The motion states and control inputs of an UAH in the 6-DOF form can be represented as follows:

$$
\begin{aligned}
& x=\{u, w, q, \theta, v, p, r, \phi, \Psi\} \\
& u_{c}=\left\{\delta_{c o l}, \delta_{\text {lat }}, \delta_{\text {lat }}, \delta_{\text {ped }}\right\}
\end{aligned}
$$

where $u, v$ and $w$ represent linear velocity in body frame; $p, q$ and $r$ denote roll, pitch and yaw rates; and $\phi, \theta$ and $\Psi$ represent the roll, pitch and yaw angle, respectively of an UAH. A single main rotor helicopter has four independent control inputs such as $\delta_{\text {lat }}, \delta_{\text {lon }}, \delta_{\text {col }}$ and $\delta_{\text {ped }}$ which denote the deflection of the lateral cyclic, longitudinal cyclic, main rotor collective pitch and tail rotor collective pitch, respectively. The collective commands control the magnitude of the main rotor and tail rotor thrust and other two control commands control the inclination of the tip-path-plane (TPP) on the longitudinal and lateral direction. The equations which describing the net forces of the UAH can be expressed as,

$$
\begin{gathered}
m(\dot{u}-v r+w q)=X-m g \sin \theta \\
m(\dot{v}-w p+u r)=Y+m g \sin \phi \cos \theta \\
m(\dot{w}+u q-v p)=Z+m g \cos \phi \cos \theta
\end{gathered}
$$

The equations which describe the moments of the UAH can be expressed as,

$$
\begin{gathered}
L=I_{x x} \dot{p}-I_{x z} \dot{r}+q r\left(I_{z z}-I_{y y}\right)-I_{x z} p q \\
M=I_{y y} \dot{q}+\operatorname{pr}\left(I_{x x}-I_{z z}\right)+I_{x z}\left(p^{2}-r^{2}\right) \\
N=-I_{x z} \dot{p}+I_{z z} \dot{r}+p q\left(I_{y y}-I_{x x}\right)+I_{x z} q r
\end{gathered}
$$

In order to complete the system modeling, the following three equations are essential which relate the Euler angle rates to the angular velocity [19].

$$
\begin{gathered}
\dot{\phi}=p+q \sin \phi \tan \theta+r \cos \phi \tan \theta \\
\dot{\theta}=q \cos \phi-r \sin \phi \\
\dot{\Psi}=\frac{q \sin \phi+r \cos \phi}{\cos \theta}
\end{gathered}
$$

The longitudinal and lateral cyclic tilt of the main rotor disk is controllable through the cyclic pitch. Therefore, the longitudinal and lateral flapping dynamics can be represented by the following first-order equations [20].

$$
\begin{aligned}
& \dot{a}_{1}=-q-\frac{a_{1}}{\tau}+\frac{1}{\tau}\left(\frac{\partial a}{\partial u} u+A_{l o n} \delta_{l o n}\right) \\
& \dot{b}_{1}=-p-\frac{b_{1}}{\tau}+\frac{1}{\tau}\left(\frac{\partial b}{\partial u} v+A_{l a t} \delta_{l a t}\right)
\end{aligned}
$$

where $\delta_{\text {lat }}$ and $\delta_{\text {lon }}$ are the lateral and longitudinal cyclic 
control inputs, al and b1 are the lateral and longitudinal flapping angles and Alon and Blat are the effective steadystate longitudinal and lateral gains from the cyclic inputs to the main rotor flapping angles. The terms $A_{u}=\frac{\partial a_{1}}{\partial u}$ and $B_{v}=\frac{\partial b_{1}}{\partial v}$ are constants and represent the longitudinal and lateral Dihedral effect. The Dihedral effect is the change of tip-path-plane (TPP) tilts due to the longitudinal and lateral velocities [21]. The Dihedral effect is modeled by the following equation.

$$
\frac{\partial a_{1}}{\partial u}=-\frac{\partial b_{1}}{\partial v}=\frac{2}{\Omega R_{b}}\left(\frac{8 C_{T}}{a \sigma}+\sqrt{\frac{C_{T}}{2}}\right)
$$

where $R_{b}$ is the main rotor radius, $\sigma$ solidity ratio, 'a' lift curve slope and $C_{T}$ thrust coefficient. Since the rotor is symmetric, so the consideration is $A_{u}=-B_{v}$. In order to design the controller, the linearization is essential to derive a simplified working model, due to the inherent instability under hover and slow flight conditions. So, after linearizing the equations (1)-(8) the following parameterized model of decoupled longitudinal and lateral dynamics can be obtained,

$$
\begin{gathered}
{\left[\begin{array}{c}
\dot{u} \\
\dot{q} \\
\dot{\theta} \\
\dot{a}_{1}
\end{array}\right]=\left[\begin{array}{cccc}
X_{u} & X_{q} & -g & X_{a} \\
M_{u} & M_{q} & 0 & M_{a} \\
0 & 1 & 0 & 0 \\
A_{u} & -1 & 0 & -\frac{1}{\tau}
\end{array}\right]\left[\begin{array}{c}
u \\
q \\
\theta \\
a_{1}
\end{array}\right]+\left[\begin{array}{c}
0 \\
0 \\
0 \\
\frac{A_{\text {lon }}}{\tau}
\end{array}\right] \delta_{\text {lon }}} \\
{\left[\begin{array}{c}
\dot{v} \\
\dot{p} \\
\dot{\phi} \\
\dot{b_{1}}
\end{array}\right]=\left[\begin{array}{cccc}
Y_{v} & Y_{p} & g & Y_{b} \\
L_{v} & L_{p} & 0 & L_{b} \\
0 & 1 & 0 & 0 \\
\frac{B_{v}}{\tau} & -1 & 0 & -\frac{1}{\tau}
\end{array}\right]\left[\begin{array}{c}
v \\
p \\
\phi \\
b_{1}
\end{array}\right]+\left[\begin{array}{c}
0 \\
0 \\
0 \\
\frac{A_{\text {lat }}}{\tau}
\end{array}\right] \delta_{\text {lat }}}
\end{gathered}
$$

where $X_{u}=\frac{1}{m} \frac{\partial X}{\partial u}, M_{u}=\frac{1}{I_{y y}} \frac{\partial M}{\partial u}$ are the force and moment derivatives normalized by the mass of the helicopter or respective moment of inertia. The pitching flap-stiffness constant is represented by $M_{a}$ that can be computed as follows $M_{a}=\frac{m M_{z}}{I_{y y}}+\frac{K_{\beta}}{I_{y y}}$, where $M_{z}$ is the height of the rotor hub above the fuselage center of gravity, Iyy is the pitching moment of inertia and $K_{\beta}$ is the rotor blade spring stiffness. Similarly the lateral flap-stiffness constant $L_{b}$ can be computed as follows $L_{b}=\frac{m M_{z}}{I_{x x}}+\frac{K_{\beta}}{I_{x x}}$. The proposed linear model as described by equations (13)-(14), has been successfully espoused for control applications in a large number of small-scale unmanned helicopters [22]-[27]. The nonlinear robust adaptive nonlinear controller will be designed using backstepping technique based on equations (13)-(14). However, before design the controller the control problem formulation is discussed in the following section.

\section{Control Problem Formulation}

From the longitudinal dynamics model as described by equation (13), it can be seen that the longitudianl flapping $a_{1}$ is a function of $u$ and $q$. Similarly, from the lateral dynamics model, it can be seen that the lateral flapping $b_{1}$ is a function of $v$ and $p$. Under this condition, to continue the design procedure of the proposed controller is not possible. From [28], it is clear that the effect of lateral and longitudinal forces produced by the flapping angles can be neglected as they have a minimal effect on the translational dynamics as compared to the propulsion forces produced by the stability derivatives $X_{\theta}$ and $X_{\varphi}$. Moreover, according to the control purposes, the dynamics of an UAH should be separated into two interconnected subsystems. The first subsystem accounts for the longitudinal dynamics and second subsystem for lateral dynamics. Now, after neglecting the effect of parameters $X_{a}, Y_{b}, X_{q}$ and $Y_{p}$ the longitudinal-lateral dynamics will have a strict feedback form which is suitable for the proposed controller. Again, as the mass of an UAH is continuously varying so the parameters of an UAH is not fixed. Moreover, the dynamics of the UAH will be affected by the external wind gusts. Thus, under the above assumptions, the simplified model equations of the longitudinal dynamics can be written as follows:

$$
\begin{gathered}
\dot{x}=u \\
\dot{u}=X_{u} u-g \theta \\
\dot{\theta}=q \\
\dot{q}=\delta M_{u} u+M_{q} q+M_{a} a_{1}+d_{1}
\end{gathered}
$$

Similarly, the simplified model equations of the lateral dynamics can be written as follows:

$$
\begin{gathered}
\dot{y}=v \\
\dot{v}=Y_{v} v+g \phi \\
\dot{\phi}=p \\
\dot{p}=\eta L_{v} v+L_{p} p+L_{b} b_{1}+d_{2}
\end{gathered}
$$

where $d_{1}$ and $d_{2}$ are the external disturbances. Based on equations (15)-(16), the proposed robust adaptive backstepping controller design procedure is shown in the following section.

\section{Controller Design}

In this section, the design procedure of the proposed controller for the longitudinal and lateral dynamics of an UAH is presented based on the appropriate decoupled model as described by equations (15)-(16). The objective of this control is to regulate the several physical quantities (e.g. position, attitude etc.) for improving the flight condition of an UAH. 


\subsection{Longitudinal Dynamics}

In this subsection, the design procedure of the adaptive robust backstepping controller is shown based on the Lyapunov function for the longitudinal dynamics as described by equation (15) under consideration of internal and external uncertainties. The design procedure is divided into four steps which is evaborately discussed as follows.

Design step 1: First, for the longitudinal position tracking objective, let define the longitudinal position tracking error as

$$
z_{1}=x-x_{d}, \dot{z}_{1}=\dot{x}-\dot{x}_{d}, \quad \dot{z}_{1}=u
$$

Here $u$ is assumed as a virtual control variable and its desired value $u_{d}$ is a stabilizing function for equation (17). Let $z_{2}$ be an another error variable representing the difference between the actual $\mathrm{u}$ and its desired value $u_{d}$, i.e.,

$$
z_{2}=u-u_{d}, \quad u=z_{2}+u_{d}
$$

Therefore, interms of $\mathrm{z} 2$ the equation (17) can be written as

$$
\dot{z}_{1}=z_{2}+u_{d}
$$

At this stage a virtual control law should be designed for ud in such a way that which would make $z_{1} \rightarrow 0$ as $t \rightarrow \infty$.

Now, consider the first CLF as follows:

$$
W_{1}=\frac{1}{2} z_{1}^{2}
$$

whose time derivative after substituting the value of $\dot{z}_{1}$ is

$$
\dot{W}_{1}=z_{1}\left(z_{2}+u_{d}\right)
$$

Now an appropriate virtual control law ud need to be selected in such a way that which would make $\dot{W}_{1} \leq 0$. Under this condition, the stabilizing function is chosen as

$$
u_{d}=-k_{1} z_{1} \text { with } \mathrm{k} 1>0
$$

where $k_{l}$ is a scalar parameter which can be used to tune the output response. Then equation (19) can be written as

$$
\dot{W}_{1}=-k_{1} z_{1}^{2}+z_{1} z_{2}
$$

From equation (21), it can be seen that if $z_{2}=0$ then

$$
\dot{W}_{1}=-k_{1} z_{1}^{2} \leq 0
$$

The second coupling term of equation (21) will be cancelled in the next step. Now the time derivative of $u_{d}$ which is essential in the next step can be written as

$$
\dot{u}_{d}=-k_{1} u
$$

As $\dot{z}_{1}=u$.

Design step 2: In this step, the error dynamics for $z_{2}=u-u_{d}$ is derived whose time derivative can be written as follows:

$$
\dot{z}_{2}=x_{u} u-g \theta+k_{1} u
$$

In which $\theta$ is viewed as an another vitual control variable. Now define a virtual control law $\theta_{d}$ and let $\mathrm{z}_{3}$ be an another error variable which is representing the difference between actual control and virtual control i.e., $z_{3}=\theta-\theta_{d}$ and after taking time derivative it can be written as

$$
\dot{z}_{2}=\left(x_{u}+k_{1}\right) u-g\left(z_{3}+\theta_{d}\right)
$$

Now choose a second CLF as follows:

$$
W_{2}=W_{1}+\frac{1}{2} z_{2}^{2}
$$

whose time derivative by inseting equations (21) and (24) can be written as

$$
\dot{W}_{2}=-k_{1} z_{1}^{2}+z_{2}\left\{z_{1}+\left(x_{u}+k_{1}\right) u-g \theta_{d}\right\}-g z_{2} z_{3}
$$

Now an appropriate stabilizing function $\theta_{d}$ can be selected in such a way to cancel out the terms related to $\mathrm{z}_{1}, \mathrm{z}_{2}$ and $\mathrm{u}$, while the term involving $\mathrm{z}_{3}$ cannot be removed and this is

$$
\theta_{d}=g^{-1}\left\{z_{1}+\left(x_{u}+k_{1}\right) u+k_{2} z_{2}\right\}
$$

Then equation (26) can be written as

$$
\dot{W}_{2}=-k_{1} z_{1}^{2}-k_{2} z_{2}^{2}-g z_{2} z_{3}
$$

From equation (28), it is clear that if $z_{3}=0$ then $\dot{W}_{2}=-k_{1} z_{1}{ }^{2}-k_{2} z_{2}{ }^{2} \leq 0$ which is negative definite. In order to complete the next step, the time derivative of $\theta_{d}$ is essential which can be written as

$$
\dot{\theta}_{d}=g^{-1}\left\{u+\left(x_{u}+k_{1}\right)\left(x_{u} u-g \theta\right)+k_{2} \dot{z}_{2}\right\}
$$

Design step 3: Here the error dynamics for $z_{3}=\theta-\theta_{d}$ is derived whose time derivative is

$$
\dot{z}_{3}=\dot{\theta}-\dot{\theta}_{d}
$$

By substituting the values of $\dot{\theta}$ and $\dot{\theta}_{d}$ into equation (30), it can be written as 


$$
\begin{aligned}
& \dot{z}_{3}=q-g^{-1}\left\{u+\left(x_{u}+k_{1}\right)\left(x_{u} u-g \theta\right)\right\} \\
& -g^{-1} k_{2}\left\{\left(x_{u}+k_{1}\right) u-g\left(z_{3}+\theta_{d}\right)\right\}
\end{aligned}
$$

In which $\mathrm{q}$ is viewed as the virtual control input. Now define a stabilizing control law qd and let $\mathrm{z} 4$ be an error variable representing the difference between actual and virtual control input, i.e., $z_{4}=q-q_{d}$ and interm of this error variable the equation (31) can be written as

$$
\begin{aligned}
\dot{z}_{3}= & z_{4}+q_{d}-g^{-1}\left\{u+\left(x_{u}+k_{1}\right)\left(x_{u} u-g \theta\right)\right\} \\
& -g^{-1} k_{2}\left\{\left(x_{u}+k_{1}\right) u-g\left(z_{3}+\theta_{d}\right)\right\}
\end{aligned}
$$

Now choose another CLF as

$$
W_{3}=W_{2}+\frac{1}{2} z_{3}^{2}
$$

whose time derivative is

$$
\begin{aligned}
\dot{W}_{3}= & -k_{1} z_{1}^{2}-k_{2} z_{2}^{2}-g z_{3} z_{2}+z_{3}\left[z_{4}+q_{d}\right. \\
& -g^{-1}\left\{u+\left(x_{u}+k_{1}\right)\left(x_{u} u-g \theta\right)\right\}-g^{-1} k_{2}\left\{\left(x_{u}+k_{1}\right) u\right. \\
& \left.\left.-g\left(z_{3}+\theta_{d}\right)\right\}\right]
\end{aligned}
$$

At this stage, the stabilizing $q_{d}$ need to be selected in such a way that cancel out the terms related to $z_{1}, z_{2}, z_{3}$ and $u$, while the term involving $z_{4}$ cannot be removed. Thus, the stabilizing function $q_{d}$ is

$$
\begin{aligned}
q_{d}= & g z_{2}+g^{-1}\left\{u+\left(x_{u}+k_{1}\right)\left(x_{u} u-g \theta\right)\right\} \\
& \left.+g^{-1} k_{2}\left\{\left(x_{u}+k_{1}\right) u-g\left(z_{3}+\theta_{d}\right)\right\}\right]-k_{3} z_{3}
\end{aligned}
$$

After that selection,

$$
\dot{W}_{3}=-k_{1} z_{1}^{2}-k_{2} z_{2}^{2}-k_{3} z_{3}^{2}+z_{3} z_{4}
$$

From equation (35), it is clear that if $z_{4}=0$ then equation (35) simplified to

$$
\dot{W}_{3}=-k_{1} z_{1}^{2}-k_{2} z_{2}^{2}-k_{3} z_{3}^{2} \leq 0
$$

In order to complete the final step, the time derivative of $q_{d}$ can be written as

$$
\dot{q}_{d}=f
$$

where

$$
\begin{aligned}
& f=g \dot{z}_{2}+g^{-1}\left\{\dot{u}+\left(x_{u}+k_{1}\right)\left(x_{u} \dot{u}-g \dot{\theta}\right)\right\} \\
& \left.+g^{-1} k_{2}\left\{\left(x_{u}+k_{1}\right) \dot{u}-g\left(\dot{z}_{3}+\dot{\theta}_{d}\right)\right\}\right]-k_{3} \dot{z}_{3}
\end{aligned}
$$

The derivation of longitudinal dynamics control law along with the stability and robustness analysis of the system is shown in the following step.

Design step 4: The dynamics of final error can be obtained as following by taking time derivative of $\mathrm{z} 4$

$$
\dot{z}_{4}=\delta M_{u} u+M_{q} q+d_{1}+M_{a} a_{1}-f
$$

In equation (38), the actual control input appears. By incorporating the estimation error of unknown parameter $\delta$, equation (38) can be rewritten as

$$
\dot{z}_{4}=(\widetilde{\delta}+\hat{\delta}) M_{u} u+M_{q} q+d_{1}+M_{a} a_{1}-f
$$

where $\widetilde{\delta}=\delta-\hat{\delta}$ is the estimation error of unknown parameter $\delta$. The objective is to design the actual control input al such that $\mathrm{z}_{1}, \mathrm{z}_{2}, \mathrm{z}_{3}$, and $\mathrm{z}_{4}$ converge to zero as $\mathrm{t} \rightarrow \infty$. The presence of the parameter estimation error suggests the following form of the CLF

$$
W_{4}=W_{3}+\frac{1}{2} z_{4}^{2}+\frac{1}{2 \gamma_{1}} \widetilde{\delta}^{2}
$$

The time derivative of $W_{4}$ becomes

$$
\dot{W}_{4}=\dot{W}_{3}+z_{4} \dot{z}_{4}-\frac{1}{\gamma_{1}} \widetilde{\delta} \dot{\hat{\delta}}
$$

where $\gamma_{1}$ is a positive design constant which determines the convergence speed of the estimation. By substituting the values of $\dot{W}_{3}$ and $\dot{z}_{4}$ into equation (41), it can be written as

$$
\begin{aligned}
& \dot{W}_{4}=-k_{1} z_{1}{ }^{2}-k_{2} z_{2}{ }^{2}-k_{3} z_{3}{ }^{2}+z_{4}\left\{z_{3}+\hat{\delta} M_{u} u+M_{q} q\right. \\
& \left.+M_{a} a_{1}-f+d_{1}\right)-\gamma_{1}^{-1} \widetilde{\delta}\left(\dot{\hat{\delta}}-\gamma_{1} M_{u} u\right)
\end{aligned}
$$

Now the final control law and adaptation law are chosen in such a way that which would make $\dot{W}_{4} \leq 0$. Thus, the final control law and adaptation law are chosen as follows:

$$
\begin{gathered}
a_{1}=-\frac{\left\{z_{3}+\hat{\delta} M_{u} u+M_{q} q-f+k_{4} z_{4}+\Gamma \operatorname{sgn}\left(z_{4}\right)+\hat{d}_{1}\right\}}{M_{a}} \\
\dot{\hat{\delta}}=\gamma_{1} z_{4} M_{u} u
\end{gathered}
$$

where $\hat{d}_{1}$ is an estimate parameter which represents a best guess for the unknown external disturbance $d_{1}$ and sgn is the signum function which can be written as

$$
\operatorname{sgn}\left(z_{4}\right)=\left\{\begin{array}{llc}
+1 & \text { if } & z_{4}>0 \\
0 & \text { if } & z_{4}=0 \\
-1 & \text { if } & z_{4}<0
\end{array}\right.
$$

The estimation error on $d_{1}$ is assumed to be bounded by knowing the constant, $\Gamma$, i.e., $\left\|d_{1}-\hat{d}_{1}\right\| \leq \Gamma$. Then by using the Schwartz inequality the derivative of W4 becomes

$\left.\dot{W}_{4} \leq-k_{1} z_{1}{ }^{2}-k_{2} z_{2}{ }^{2}-k_{3} z_{3}{ }^{2}-k_{4} z_{4}{ }^{2}-\left\|z_{4}\right\| \mid \Gamma-\left\|d_{1}-\hat{d}_{1}\right\|\right]$

Since $\left\|d_{1}-\hat{d}_{1}\right\| \leq \Gamma$, so $\dot{W}_{4} \leq 0$. 


\subsection{Lateral Dynamics}

In this subsection, the robust adaptive backstepping controller is designed based on the Lyapunov function for the lateral dynamics in the presence of parametric and external uncertainties. Again, consider a CLF which is used to augment the estimated parameter error,

$$
W_{10}=W_{7}+\frac{1}{2} z_{8}^{2}+\frac{1}{2 \gamma_{l}} \widetilde{\eta}^{2}
$$

Using the similar procedure as mentioned in the previous subsection, the following control input can be obtained for the lateral dynamics

$$
\begin{gathered}
b_{1}=-L_{b}^{-1}\left\{z_{7}+\hat{\eta} L_{v} v+L_{p} p-f\left(z_{6}, z_{7}, v, \phi, \phi_{d}\right)+\right. \\
\left.k_{8} z_{8}+\Gamma_{1} \operatorname{sgn}\left(z_{8}\right)+\hat{d}_{2}\right\} \\
\dot{\hat{\eta}}=\gamma_{2} L_{v} v z_{8}
\end{gathered}
$$

Finally, we get the following equation.

$$
\dot{W}_{10}=-k_{5} z_{5}^{2}-k_{6} z_{6}^{2}-k_{7} z_{7}{ }^{2}-k_{8} z_{8}^{2} \leq 0
$$

Thus, it can be proved that the system is Lyapunov stable and the errors are asymptotically converging to an arbitrarily small neighborhood of zero. Note that the detailed design procedure of the proposed controller for lateral dynamics is not illustrated in this paper. Simulation studies are conducted in the following section to show the effectiveness of this proposed controller.

Remark 1: Control inputs in the controller design process are set to be longitudinal and lateral flapping angles. They will be converted later into longitudinal cyclic and lateral cyclic for implementation.

\section{Simulation Results}

The performance of the designed controller has been conducted on a nonlinear simulation model using the MATLAB simulink. To show the superiority of the proposed robust adaptive backstepping controller over an existing controller, the performance is also compared with a classical PID controller. The simulation is conducted in the case where the desired positions are set to $\mathrm{x}=0 \mathrm{~m}$ and $\mathrm{y}=0 \mathrm{~m}$. The roll angle trim $\phi$ ref is initialized at 4.50 to compensate the effect of tail rotor thrust.

The corresponding system responses with both controllers are shown in Fig. 1 to Fig. 3. The horizontal position responses of an UAH with both controllers is shown in Fig. 1. It is clear that the position tracking error is almost zero with the proposed controller (solid blue line) but it is relatively large with the PID controller (solid green line) and it is oscillating.

From the simulation result, it is clear that the proposed controller is more robust than the PID control under the condition of parametric and external disturbances within the system of an UAH. The corresponding velocity response of an UAH is shown in Fig. 2, from where it is clear that horizontal velocities settle to approximately $0 \mathrm{~m} / \mathrm{s}$ at about $3 \mathrm{~s}$ after the start of the simulation in both $y$ direction and $x$ direction from the beginning of the simulation. But for the PID controller, it can be seen that they are oscillating and are not completely damped.

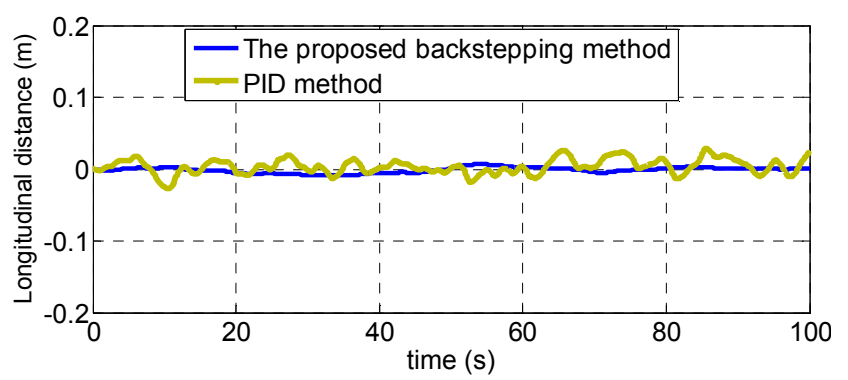

(a). Longitudinal distance of UAH.

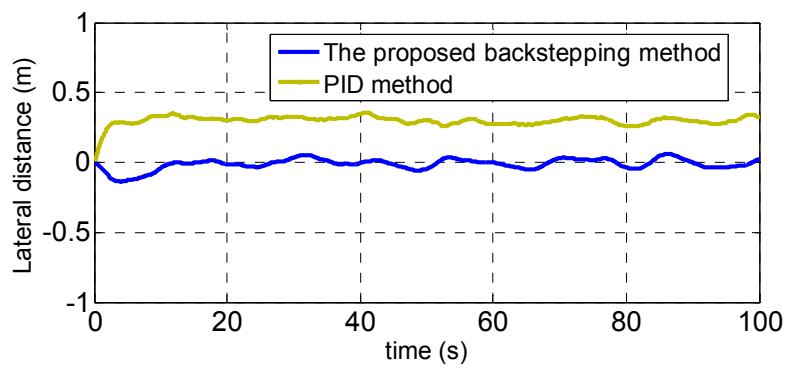

(b). Lateral distance of UAH.

Fig. 1. Horizontal positions response using robust adaptive backstepping and PID controllers.

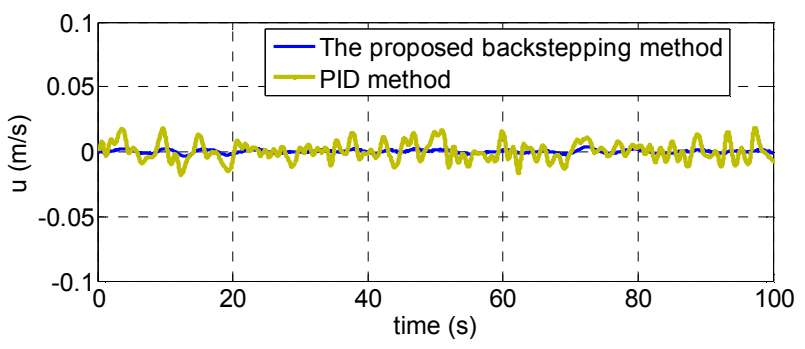

(a). Longitudinal velocity of UAH.

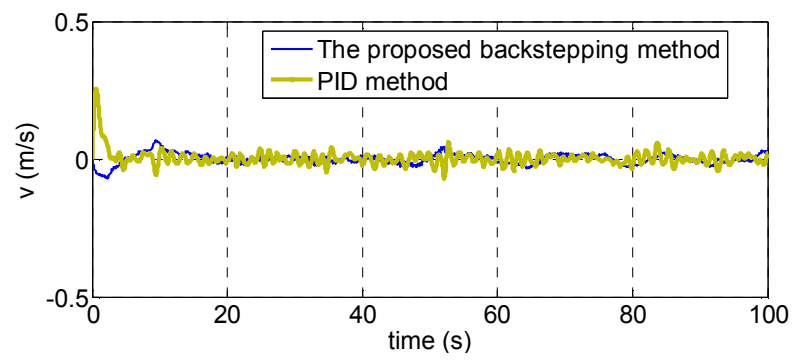

(b). Lateral velocity of UAH.

Fig. 2. Horizontal velocities response using robust adaptive backstepping and PID controllers.

Thus, from the simulation results, it is obvious that the proposed controller is able to achieve the desired horizontal 
positions in the presence of parametric and external disturbances by providing more stabilize hovering flight.

The corresponding roll and pitch angle responses of an UAH during hover flight are shown in Fig. 3. From where, it can be seen that due to the proposed controller, the roll angle settles to the desired value at $4.5^{\circ}$ wihtin few second, but for the PID controller, it settles in between about $2.5^{\circ}$ to $3.90^{\circ}$. It can be seen that the angle responses are more stable with the proposed controller than the existing controller in terms of settling time and oscillations.

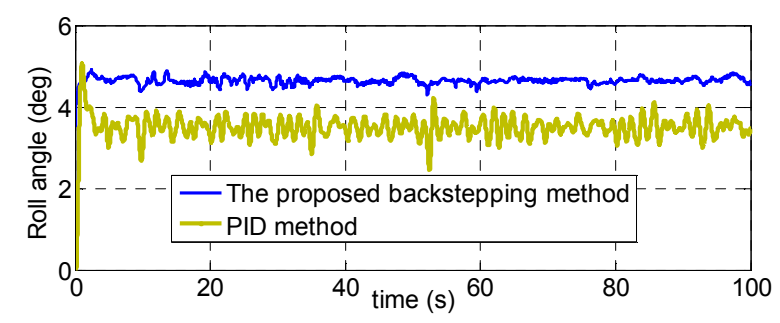

(a). Roll angle of UAH.

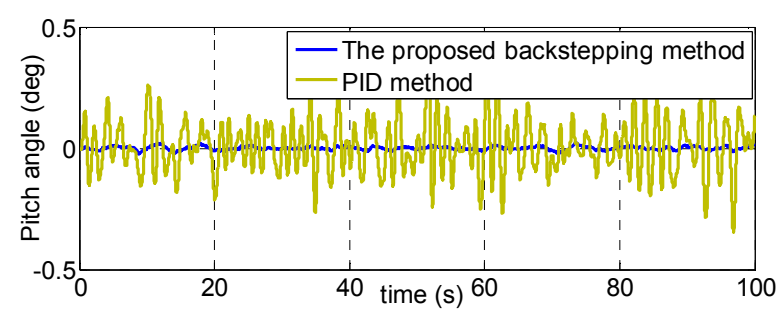

(b). Pitch angle of UAH.

Fig. 3. Roll and Pitch angles response using the adaptive robust backstepping and PID controllers.

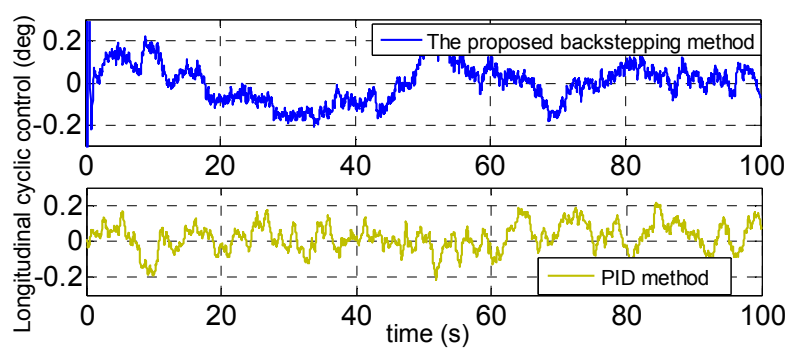

(a). Control signal of longitudinal dynamics.

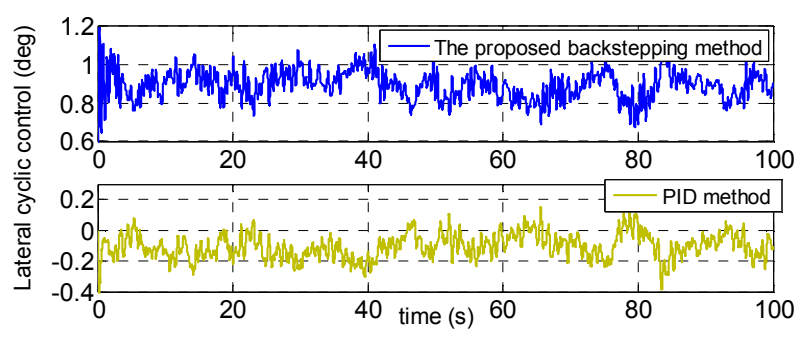

(b). Control signal of lateral dynamics.

Fig. 4. Control signals using the robust adaptive backstepping and PID controllers.

The control signals for the both controllers is shown in Fig. 4, from where it can be seen that control signals do not exceed the physical constraints of the helicopter. From the simulation results, it is clear that the designed controller is more effective than an existing controller in terms of settling time and damping of oscillation.

\section{Conclusion}

In this paper, a backstepping method to design a robust adaptive controller for an UAH is proposed to enhance the stabilization of horizontal position control of a hovering flight. Based on the proposed formulation, the designed controller is adaptive to the unknown parameters and robust to the external disturbances. From the theoretical and numerical evaluations, it can be concluded that the proposed controller has the capability to stabilize the longitudinal and lateral dynamics as it can track the pre-defined reference trajectory despite the presence of parametric and external uncertainties within the UAH system model. Future work will be devoted on the implemention of the controller to a real system, e.g., flight test under the consideration of parametric and external uncertainties to prove the feasibility in the real life.

\section{References}

[1] T. K. Roy, M. Garrat, H. R. Pota and H. Teimoori, "Hover flight control of a small helicopter using robust backstepping and PID," in Proceedings of the 10th World Congress on Intelligent Control and Automation, 6-8 July 2012.

[2] L. Guo, C. Melhuish, and Q. Zhu, "Towards neural adaptive hovering control of helicopters," in Proc. IEEE Int. Conf. Control Applications, Glasgow, U.K., Sep. 2002, pp. 54-58.

[3] T. K. Roy, "Position control of a small helicopter using robust backstepping," in Proceedings of the IEEE 7th International Conference on Electrical and Computer Engineering, pp. 787790, 20-22 December 2012.

[4] T. K. Roy and A. A. Suman, "Adaptive backstepping controller for altitude control of a small-scale helicopter by considering the ground effect compensation," in Proc. of the IEEE2nd International Conference on Informatics, Electronics and Vision (ICIEV), pp. 1-5, 2013, Bangladesh.

[5] H. Shim, T. J. Koo, and F. Hoffmann, "A comprehensive study of control design for an autonomous helicopter," in Proceedings of the $37^{\text {th }}$ International Conf. on Decision and Control, pp. 3653-3658, 1998.

[6] X. H. Xia, and Y. J. Ge, "Finite-horizon optimal linear control for autonomous soft landing of small-scale helicopter," In Proceedings of the International Conf. on Information and Automation, pp. 1160-1164, 2010.

[7] T. K. Roy, and M. Garrat, "Altitude control of an unmanned autonomous helicopter via robust backstepping controller under horizontal wind gusts," in Proceedings of the IEEE 7th International Conf. on Electrical and Computer Engineering, pp. 771-774, 20-22 December 2012.

[8] T. K. Roy, M. Garrat, H. R. Pota, and H. Teimoori, "Robust altitude control of an unmanned autonomous helicopter using backstepping," in Proceedings of the 10th WCICA, pp. 16501654, 6-8 July 2012. 
[9] T. K. Roy and H. M. Rasel, "Altitude control of an unmanned autonomous helicopter via adaptive backstepping controller under horizontal wind gusts," IJCEE, vol. 5, no. 1, pp. 1-7, 2013.

[10] T. K. Roy and A. A. Suman, "Adaptive backstepping controller for altitude control of a small-scale helicopter by considering the ground effect compensation," in Proc. of the IEEE2nd International Conference on Informatics, Electronics and Vision (ICIEV), May 2013, pp. 1-5, Bangladesh.

[11] T. K. Roy, M. A. Mahmud, M. Z. I. Sarkar, and M. F. Pervej, "Robust backstepping controller for yaw dynamics control of an unmanned autonomous helicopter," in Proc. of the International Forum on Strategic Technology (IFOST), 2014.

[12] D. J. Wallcer, M.C. Turner, AJ. Smerlas, ME. Strange, and A.W. Gubbels, "Robust control of the longitudinal and lateral dynamics of the BELL 205 helicopter," Proceedings of the American Control Conference, June 1999.

[13] K. A. Danapalasingam, J.-J. Leth, A. la C.-Harbo and M. Bisgaard "Robust helicopter stabilization in the face of wind disturbance" 49th IEEE Conference on Decision and Control, December 15-17, 2010.

[14] T. Cheviron, F. Plestan, and A. Chriette, "A robust guidance and control scheme of an autonomous scale helicopter in presence of wind gusts," International Journal of Control, Vol. 82, No. 12, December 2009

[15] T. K. Roy, M. Garrat, H. R. Pota and H. Teimoori, "Robust control for longitudinal and lateral dynamics of small scale helicopter, in Proceedings of the $31^{\text {st }}$ CCC, 25-27 July, 2012.

[16] S. Suresh, P. Kashyab and M. Nabi, "Automatic take-off control system for helicopter an $\mathrm{H} \infty$ apporach," 11th Conf. Control, Automation, Robotics and Vision, 7-10 December, 2010.

[17] A. Isidori, L. Marconi and A. Serrani, "Robust nonlinear motion control of a helicopter," IEEE Transactions on Automatic Control, Vol. 48, No. 3 March 2003.

[18] L. Guo, C. Melhuish, and Q. Zhu, “Towards neural adaptive hovering control of helicopters," in Proc. IEEE Int. Conf. Control Applications, Glasgow, U.K., Sep. 2002, pp. 54-58.

[19] D. McLean. Automatic Flight Control Systems. Prentice Hall, 1990.

[20] T. K. Roy, "Robust adaptive control for longitudinal and lateral dynamics of a small scale helicopter," IJCCNet, vol. 1, no. 3 , pp. $22-34,2012$.

[21] T. K. Roy, "Robust backstepping control for small helicopter" Master thesis, November 2012, The University of New South Wales, Australia.

[22] M. Tischler and R. Remple, "Aircraft and rotorcraft system identification: engineering methods with flight-test examples," in AIAA, 2006

[23] T. K. Roy, "Horizontal position control of a small scale helicopter using adaptive backstepping controller," IJCCNet, vol. 2, no. 2, pp. 1 - 14, May 2013.

[24] T. K. Roy, M. Garrat, H. R. Potat and H. Teimoori, "Robust Backstepping Control for Longitudinal and Lateral Dynamics of Small Scale Helicopter," Journal of University of Science and Technology of China, Vol. 42, No. 7, Jul. 2012.

[25] T. K. Roy, "Longitudinal and lateral dynamics control of a small scale helicopter using adaptive backstepping controller under horizontal wind gusts," Asian Transaction Engineering, vol. 3, no. 1, March 2013.

[26] G. Cai, B. Chen, K. Peng, M. Dong, and T. Lee, "Modeling and control system design for a UAV helicopter," in 14th Mediterranean Conference on Control and Automation, June 2006, pp. 1-6.

[27] T. K. Roy, "Longitudinal and lateral dynamics control of a small scale helicopter using adaptive backstepping controller under horizontal wind gusts," Asian Transaction on Engineering, vol. 3, no. 1., 2013.

[28] I. A. Raptis and K. P. Valavanis, "Velocity and heading tracking control for small-scale unmanned helicopters," in Proceedings of American Control Conference, 2011. 